\title{
Influence of Chemical Treatment on Thermal Decomposition and Crystallite Size of Coir Fiber
}

\author{
R. Manjula ${ }^{1}$ - N. V. Raju ${ }^{1}$ - R. P. S. Chakradhar ${ }^{2}$. \\ Ekwipoo Kalkornsurapranee $^{3}$ • Jobish Johns ${ }^{4}$
}

Received: 6 March 2017 / Accepted: 31 October 2017 / Published online: 20 November 2017

(C) Springer Science+Business Media, LLC 2017

\begin{abstract}
Coir fibers were treated with sodium hydroxide $(\mathrm{NaOH})$ and glutaraldehyde (GA). The influence of alkali and aldehyde treatment on thermal degradation and crystallinity of coir fiber was studied in detail. Thermogravimetric analysis and X-ray diffraction techniques were mainly used to characterize the coir samples. Activation energy of degradation was calculated from Broido and Horowitz-Metzger equations. $\mathrm{NaOH}$-treated samples showed an increase in thermal stability. Removal of impurities such as waxy and fatty acid residues from the coir fiber by reacting with strong base solution improved the stability of fiber. Crosslinking of cellulose with GA in the fiber enhanced the stability of the material. Scanning electron microscopy was employed to analyze the change in surface morphology upon chemical treatment. Improvement in the properties suggests that $\mathrm{NaOH}$ and GA can be effectively used to modify coir fiber with excellent stability.
\end{abstract}

Keywords Activation energy · Coir fiber · Crystallinity · Glutaraldehyde · Thermal stability

Jobish Johns

jobish_johns@rediffmail.com

1 Department of Physics, Global Academy of Technology, Bangalore-98, India

2 Surface Engineering Division, CSIR-NAL, Bangalore -17, India

3 Department of Materials Science and Technology, Faculty of Science, Prince of Songkla University, Hat-Yai, Thailand

4 Department of Physics, Rajarajeswari College of Engineering, Bangalore-74, India 


\section{Introduction}

Plant fibers are also called as lignocellulosic fibers, which include bast fibers, leaf fibers, fruit fibers and other grass fibers. They are rich in lignin, cellulose, hemicelluloses and can be used for various applications such as ropes, yarns. As a substitute for the declining supply of fossil fuel resources, biomass has been widely used as reinforcing fillers in composites [1]. These composites gained attention due to its application in engineering such as construction materials and structural parts of vehicles where reduction in weight is required [2]. Such composites also contribute to the reduction in pollution, global warming and waste generation [3]. These fiber composites are suitable for its relatively high strength and stiffness, low cost, low density and renewability. However, limitations exhibited by the plant fibers are moisture absorption and low thermal stability. This degradation is influenced by its chemical structure which includes lignin, hemicelluloses and cellulose. Coir decomposition rate is relatively low which may be due to higher lignin content.

Coconut fiber is a fruit fiber extracted from outer shell of a coconut. Coconut is a tropical fruit mainly cultivated in tropical countries such as India, Sri Lanka, Brazil, Thailand, Vietnam, Philippines and Indonesia [4]. There are two types of coconut fiberBrown fiber extracted from mature fruit and white fibers extracted from immature fruit. Brown fiber can be used in engineering since it is mechanically strong and having high abrasion resistance. Mature brown fiber contains more lignin and less cellulose [5].

Lignocellulosic coir fibers are the potential biodegradable and renewable material which is used to make composites for various applications such as insulation, packaging, automobile parts, construction. It is essential to do some chemical modifications to achieve better physical properties and thermal stability. It leads to improve the strength of the resulting composite material. Plant fibers generally degrade thermally through degradation, depolymerization and oxidation when it is heated. For example, degradation of hemicelluloses takes place at $300^{\circ} \mathrm{C}$, whereas cellulose degrades at $350^{\circ} \mathrm{C}$ and lignin degrades between $250^{\circ} \mathrm{C}$ and $600^{\circ} \mathrm{C}$ [6]. This is due to the breakdown of $\beta$-1-4-glycosidic chains from celluloses and hemicelluloses as well as cleavage of $\beta$-O-4-alkyl-aryl ether linkages from lignin [7].

The processing conditions have a major role to improve the mechanical properties of the composites by enhancing the interaction between matrix and reinforcements [2,8-14]. The effect of heating and aging on the mechanical properties of fibers can be studied by exposing the fibers at different temperatures before the preparation of composites. Alkali treatment is an economical method to improve the adhesivity between fiber and matrix. The strength deterioration during the alkali treatment is the main drawback [15].

Chemical treatment using GA is now introduced and included in this paper. Zefang Xiao et al. studied the effect of GA on the properties of wood [16]. It has been reported that GA reduces the water uptake and moisture absorption and enhances the resistance of decay organisms because of the ability to block hydroxyl groups [17]. Limited information is available in the literature regarding the influence on physical properties of wood or plant fibers with GA treatment.

The aim of this study is to investigate the effect of chemical treatment on the thermal stability of coir fibers. It is appropriate to study the thermal stability in terms of 
decomposition of coir fibers to be used for the preparation of composites. Chemical modification of coir fibers improves the surface topology, wettability and mechanical strength. Hence, the thermal stability of these fibers is also expected to increase compared to raw fibers. This may be due to the change in its microstructure $[18,19]$. Incorporation of such type of fillers may be effectively influence on improving the stability of resulting composites.

\section{Materials and Methods}

\subsection{Materials}

Coir fibers which were procured from Central Institute of Coir technology, Bangalore, were used for study. These fibers were extracted from coconut husk of mature fruit known as brown fibers and were extracted by process such as decorticators and used as received. The moisture content of the sample was about $7.74 \%$. NaOH pellets, $25 \%$ aqueous $\mathrm{G}$ and phenolphthalein indicator was procured from Karnataka Fine Chem, Bangalore. The chemical composition of coir used for the studies is lignin $43.61 \%$, cellulose $39.79 \%$, hemicellulose $5 \%$, pectin $0.95 \%$ and ash $3.9 \%$.

\subsection{Experimental}

Alkali treatment has been done for pure fibers by immersing in $\mathrm{NaOH}$ solution. A $50 \%$ (w/w) $\mathrm{NaOH}$ solution was prepared initially by dissolving $500 \mathrm{gm}$ of $\mathrm{NaOH}$ in $500 \mathrm{ml}$ distilled water. Then, the coir sample was immersed in $\mathrm{NaOH}$ solution for 2 hours and washed with distilled water to remove $\mathrm{NaOH}$ completely from the fiber. The drained water was checked for neutrality by adding phenolphthalein as an indicator. The washing was continued till there was no change in color of drained water. These fibers were dried in oven at $50{ }^{\circ} \mathrm{C}$ for 2 hours and introduced for tensile testing. In addition to alkali treatment, the fibers were introduced for aldehyde treatment. An aqueous solution of GA ( $25 \%$ ) was used for the chemical modification of fiber. Coir fibers were immersed in GA for one day and dried in oven at $50{ }^{\circ} \mathrm{C}$ for 2 hours. Then, the resulting samples were introduced for XRD and thermal analysis.

\subsection{X-ray Diffraction (XRD)}

X-ray diffractograms were collected using a Bruker AXS D8 diffractometer with a monochromatic $\mathrm{K}_{\alpha} \mathrm{Cu}$ radiation of wavelength $1.5406 \AA$. Intensities were measured in the range of $5^{\circ}$ to $85^{\circ}$, and $\mathrm{Si}(\mathrm{Li}) \mathrm{PSD}$ was used as the detector. d spacing was calculated using Braggs equation.

Crystallinity index was determined using the empirical formula proposed by Segal et al. [19]

$$
C I=\frac{\left(I_{002}-I_{a m}\right) \times 100}{I_{002}}
$$


where $\mathrm{I}_{002}$ is the maximum intensity of (200) lattice diffraction and $\mathrm{I}_{\mathrm{am}}$ is the intensity of diffraction of amorphous band.

The apparent size of the crystallite was calculated using Scherrer equation [19-21]

$$
D=\frac{K \lambda}{\beta \cos \theta}
$$

where $\mathrm{K}$ is Scherrer constant (0.94), $\lambda$ is the wavelength of X-rays used, $\beta$ is the full width at half maxima of the diffraction band and $\theta$ is the Braggs angle corresponding to (200) plane.

\subsection{Fourier Transform Infrared Spectroscopy (FTIR)}

Fourier transform infrared spectroscopy studies on pristine and chemically treated fibers were carried out by dispersing the powdered fiber samples on $\mathrm{KBr}$ pellets and using a Thermo Nicolet, Avatar 370 FTIR spectrophotometer. All the fiber samples were recorded in the $4000-400 \mathrm{~cm}^{-1}$ region with 32 scans in each case at a resolution of $4 \mathrm{~cm}^{-1}$.

\subsection{Thermogravimetric Analysis (TGA)}

Thermogravimetric analysis was performed using PerkinElmer Diamond TG/DTA analyzer in nitrogen atmosphere with a flow rate $20^{\circ} \mathrm{C} / \mathrm{min}$ from $25^{\circ}$ to $700^{\circ} \mathrm{C}$. Approximately $10 \mathrm{mg}$ of sample was analyzed. Broido and Horowitz-Metzger equations [22,23] were used to calculate the activation energy of degradation (Table 1).

\section{Results and Discussion}

Crystalline nature of any solid can be analyzed by X-ray diffraction (XRD) method. Degree of crystallinity of coir fiber samples is determined using XRD. In plant fibers, cellulose is crystalline, whereas lignin and hemicellulose are non-crystalline. The intramolecular and intermolecular hydrogen bonds are formed due to the presence of free hydroxyl group in cellulose which gives rise to ordered crystalline arrangement.

Figure 1 shows the XRD diffractograms of raw and chemically modified fibers. The graph shows peaks $(2 \theta)$ around $16.5^{\circ}, 22^{\circ}$ and $34.6^{\circ}$ which represent the typical cellulose I structure and these peaks are due to (110), (200) and (004), respectively $[24,25]$. Crystallinity index is also calculated using Equation 1, and it is found to be

Table 1 Kinetic methods used in evaluating activation energy

\begin{tabular}{lll}
\hline Method & Expressions & Plot \\
\hline Broido & $\ln (\ln (1 / y))=\frac{E}{R T}+$ Cons $\tan t$ & $\ln (\ln (1 / y))$ against $\frac{1}{T}$ \\
Horowitz-Metzger & $\ln (-\ln (1-\alpha))=-E \theta / R T^{2}+$ Cons $\tan t$ & $\ln (-\ln (1-\alpha))$ against $\theta$ \\
\hline
\end{tabular}




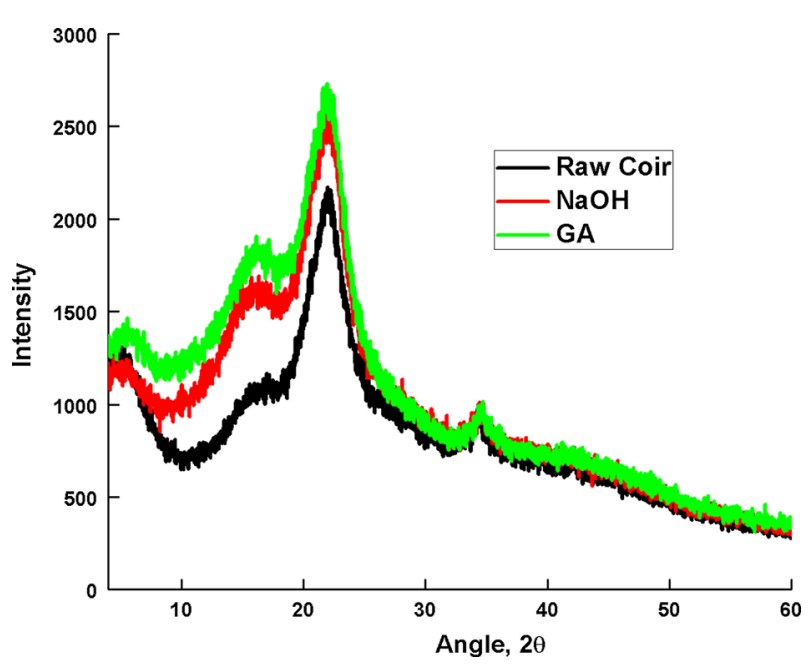

Fig. 1 XRD diffractograms for raw, NaOH-treated and GA-treated fibers

Table 2 Parameters obtained from XRD analysis of coir samples

\begin{tabular}{lllll}
\hline & $2 \theta\left({ }^{\circ}\right)$ & $\mathrm{d}(\AA)$ & $\mathrm{CI}(\%)$ & $\mathrm{D}(\mathrm{nm})$ \\
\hline Raw fiber & 22.12 & 4.0149 & 50.8 & 3.98 \\
NaOH-treated fiber & 22.13 & 4.013 & 39.57 & 3.16 \\
GA-treated fiber & 22.18 & 4.00 & 36 & 2.78 \\
\hline
\end{tabular}

$50.8 \%$ for the raw fiber, $39.51 \%$ for $\mathrm{NaOH}$-treated fiber and $34.04 \%$ for the fibers treated with GA. Generally, cellulose is crystalline in nature among polymers. A small percentage of disorder ness can be found in case of cellulose. Crystallinity index (CI) has been used to describe the relative amount of crystalline portion in cellulose. Decrease in crystallinity index may be due to the reorganizing molecular arrangement or due to partial dissolving of the crystalline parts when treated with alkali and aldehyde (Table 2).

Crystallite size was calculated using Equation 2, and it is found that raw fibers have a higher crystallite size as compared to $\mathrm{NaOH}$ - and GA-treated fibers as shown in Table 2. Rearrangement or shrinking might be occurred during $\mathrm{NaOH}$ and GA treatment on coir fibers.

FTIR peaks can also be used to analyze crystallinity of samples containing cellulose I or II or mixture of both and amorphous cellulose. Figure 2 shows the FTIR spectra of raw, NaOH-treated and GA-treated coir fiber. The region $800 \sim 1500 \mathrm{~cm}^{-1}$ is sensitive to crystalline structure of cellulose. Spectral bands at $1420 \sim 1430 \mathrm{~cm}^{-1}$ and $893 \sim 897 \mathrm{~cm}^{-1}$ are used to determine the crystal structure of cellulosic material [2426].

The spectral ratio $(1420 / 893) \mathrm{cm}^{-1}$ shows index of crystallinity or lateral order index (LOI) and spectral ratio $(1375 / 2900) \mathrm{cm}^{-1}$ shows total crystallinity index (TCI) (Table 3). LOI is correlated with the overall degree of order in the cellulose, and TCI is proportional to the crystallinity degree of cellulose. From the values of LOI and 


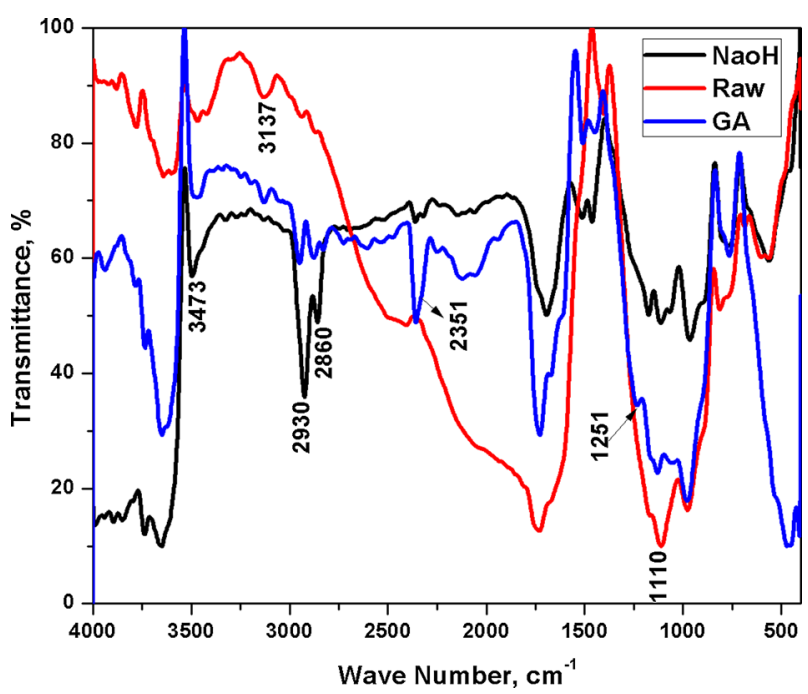

Fig. 2 FTIR spectra of untreated, NaOH-treated and GA-treated coir fibers

Table 3 TCI, LOI and HBI values of raw, $\mathrm{NaOH}$-treated and GA-treated fibers

\begin{tabular}{llll}
\hline Samples & LOI & TCI & HBI \\
\hline Raw & 0.0835 & 0.408 & 0.377 \\
NaOH-treated fiber & 0.429 & 0.285 & 1.263 \\
GA-treated fiber & 0.178 & 0.5062 & 0.597 \\
\hline
\end{tabular}

TCI, the nature of crystallinity of the material can be determined. The hydrogen bond intensity (HBI) of cellulose is closely related to the amount of bound water, the degree of intermolecular regularity or crystallinity calculated considering the chain mobility and bond distance (Table 3). The spectral ratio between the absorbance bands at $3400 \mathrm{~cm}^{-1}$ and $1320 \mathrm{~cm}^{-1}$ was used to study the HBI of the fibers [6].

LOI is low for raw as compared to the fibers treated with $\mathrm{NaOH}-$ and GA-treated fibers. This may be due to the removal of amorphous cellulose. TCI is higher for GAtreated fibers and least for $\mathrm{NaOH}$-treated fibers. It shows that fiber treated with $\mathrm{NaOH}$ has higher degree of order, whereas cellulose shows lower degree of crystallinity. Fibers treated with GA show an increase in degree of crystallinity attributed to the presence of cellulose and lower degree of order. Ornaghi et al observed that the higher extractive content can show higher values in the specific band [25]. The band at $2900 \mathrm{~cm}^{-1}$ is associated with linear chain extractives derived by hydrocarbonates. Higher extractive content leads to lower in the total crystallinity value as observed in the fibers treated with $\mathrm{NaOH}$. The ratio of (3400/1320) in FTIR spectrum represents the amount of bound water in the fiber structure. The ratio is higher for $\mathrm{NaOH}$-treated fibers indicating more amount of bound water in the fiber than that of fibers treated with GA. It clearly indicates higher degree of crystallinity of cellulose in $\mathrm{NaOH}$-treated fibers compared to the raw fibers and fibers treated with GA. 


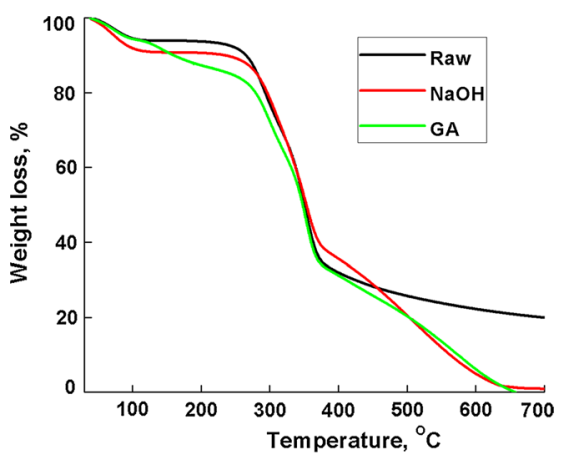

(a)

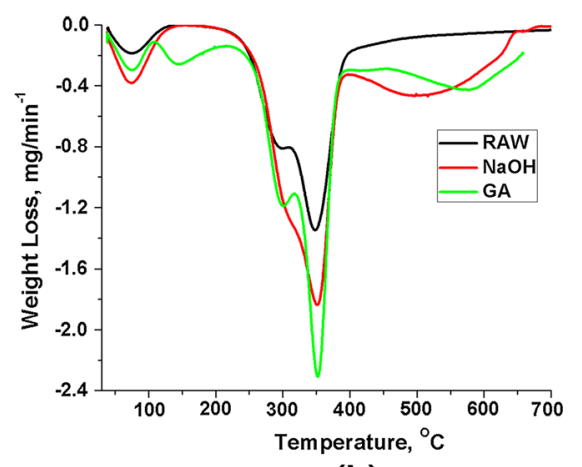

(b)

Fig. 3 (a) DTA (b) DTG curves for raw coir, fibers treated with $\mathrm{NaOH}$ and GA-treated fibers

Strong bands are observed at $3137 \mathrm{~cm}^{-1}, 2930 \mathrm{~cm}^{-1}$ and $2860 \mathrm{~cm}^{-1}$ which corresponds to the stretching of alkyl group of cellulose as well as lignin present in the coir fiber. The band at $1110 \mathrm{~cm}^{-1}$ is representing carboxyl group of fatty acids present in untreated coir fiber. In case of $\mathrm{NaOH}$-treated fibers, the peak corresponds to carboxyl group which is almost disappeared. It indicates the removal of fatty acids from coir fiber by alkali treatment. The intensity of peak at $3473 \mathrm{~cm}^{-1}$ corresponds to hydroxyl group also increased for $\mathrm{NaOH}$-treated fibers. The appearance of peak at $1251 \mathrm{~cm}^{-1}$ in case of GA-treated fibers can be attributed to the crosslinking reaction of cellulose and lignin. This may be due to the formation of ether groups during crosslinking in the presence of GA. The crosslinking of cellulose can also be confirmed from the band $2351 \mathrm{~cm}^{-1}$ due to $C-H$ deformation.

Studying the thermal stability of fibers used as filler to fabricate polymer composites is most significant [26]. Reinforcement of fibers in the polymer matrix is used to carry out at temperatures around $200^{\circ} \mathrm{C}$. Degradation of fibers due to high temperatures at the time of processing may lead to reduce the efficiency of resulting composites. Changes consist of odor, browning and reduction in mechanical properties of the fibers. Hence, it is necessary that the degradation profile of the fibers must be determined prior to their use in composite applications [3]. The thermogravimetric curves for the coir fibers studied are presented in Fig. 3. There is a considerable change in the degradation behavior of untreated and chemically modified coir fibers. Raw fiber shows slow degradation when compared to $\mathrm{NaOH}$ - and GA-treated fibers. The presence of fatty acids and waxy residues present in the raw fiber is responsible for higher thermal stability compared to the chemically treated fibers.

Table 4 shows the temperature at different weight losses for raw and chemically modified fibers. Weight loss can be seen around $70^{\circ} \mathrm{C}$ which is due to the loss of bound water. Thermal degradation of coir fiber is a three-step process where the first degradation starts at around $300^{\circ} \mathrm{C}$ due to hemicelluloses. The second stage is due to the degradation of cellulose at $350^{\circ} \mathrm{C}$. Slow degradation of lignin takes place between $180^{\circ} \mathrm{C}$ and $350{ }^{\circ} \mathrm{C}$. The chemical structure of hemicelluloses is attributed to the higher activity in thermal decomposition. Hemicellulose has a random amorphous structure and can be easily hydrolyzed as compared to cellulose which is very long and 
Table 4 Temperature at different weight losses $\left({ }^{\circ} \mathrm{C}\right)$ for raw and chemically modified fibers

\begin{tabular}{lllllllllll}
\hline Samples & \multicolumn{1}{l}{ Temperature at different weight losses $\left({ }^{\circ} \mathrm{C}\right)$} \\
\cline { 2 - 11 } & $5 \%$ & $10 \%$ & $15 \%$ & $20 \%$ & $25 \%$ & $30 \%$ & $35 \%$ & $40 \%$ & $45 \%$ & $50 \%$ \\
\hline Raw & 92.75 & 265.5 & 282.3 & 293.4 & 304.7 & 317.4 & 329.1 & 338.0 & 344.9 & 351.0 \\
NaOH-treated & 75.13 & 233.3 & 281.6 & 296.7 & 307.6 & 318.4 & 328.5 & 337.7 & 345.8 & 353.5 \\
GA-treated & 89.7 & 159.78 & 243.05 & 279.54 & 293.8 & 305.8 & 318.5 & 333.3 & 340.8 & 348.1 \\
\hline
\end{tabular}

crystalline. This increases the thermal stability of fibers. Lignin has high molecular weight and composed of three kinds of benzene-propane units. Therefore, the thermal stability of lignin is very high, and it cannot be easily decomposed [27].

Higher thermal stability is associated with higher total crystallinity index and crystalline size of cellulose for the fibers treated with $\mathrm{NaOH}$. Table 5 shows raw fiber has higher values in residue weight of $22.23 \%$ at $600^{\circ} \mathrm{C}$ and $\mathrm{T}_{\mathrm{i}}$. This may be due to the higher inorganic content present in raw fibers. Fibers treated with $\mathrm{GA}$ and $\mathrm{NaOH}$ have lower residue weight which may be due to the removal of inorganic content. Water content in the fiber increases with $\mathrm{NaOH}$ treatment. Bound water evaporates, and weight loss starts at a relatively low temperature.

Decomposition of cellulose and hemicellulose occurs in different stages. The peaks partially overlap each other and appears as a more or less pronounced shoulder instead of a well-defined peak. For $\mathrm{NaOH}$-treated fibers, the shoulder appears at a higher temperature compared to the other fibers. This may be a consequence of lesser removal of volatile contents and lower hemicellulose reactivity.

Major weight loss at high temperature is mainly due to the degradation of cellulose which is evident from the DTG peaks for the fibers treated with $\mathrm{NaOH}$ and GA. For GA-treated fibers, the main degradation of cellulose occurs at $351.86^{\circ} \mathrm{C}$ (Table 4). Formation of crosslinked cellulose on treating the fiber with GA delays decomposition.

Activation energy is defined as the least amount of energy required to decompose 1 mole of a substance, and it reveals the information about thermal stability. Activation energy of degradation has been calculated using two methods. Larger activation energy indicates higher stability of the material. As coir fibers are used to fabricate composite materials, no absolute value of activation energy can be expected. In this study, activation energy is calculated using Broido and Horowitz-Metzger equations. From both the equations, higher activation energy valued can be seen for fibers treated with $\mathrm{NaOH}$ and GA-treated fibers. Uncured coir fiber shows the least activation energy. Removal of waxy and fatty acids from the fiber during $\mathrm{NaOH}$ treatment improves the activation energy. Crosslinking of cellulose and lignin present in the fiber through GA curing may be the reason to enhance the activation energy.

The surface morphology of coir fiber before chemical treatment is shown in Fig. 4a. A rough surface with irregular strips can be seen in the SEM microphotograph of uncured coir fiber. The fiber is covered with a layer of impurities including lignin. Roughened surface is observed for the coir fiber after the alkali treatment with $\mathrm{NaOH}$ (Fig. 4b). Many pits are formed on the surface of alkali treated fibers due to the 


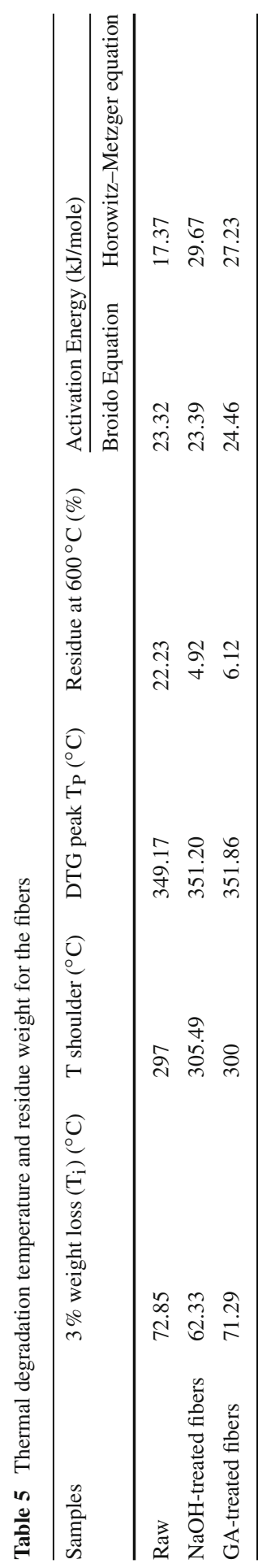



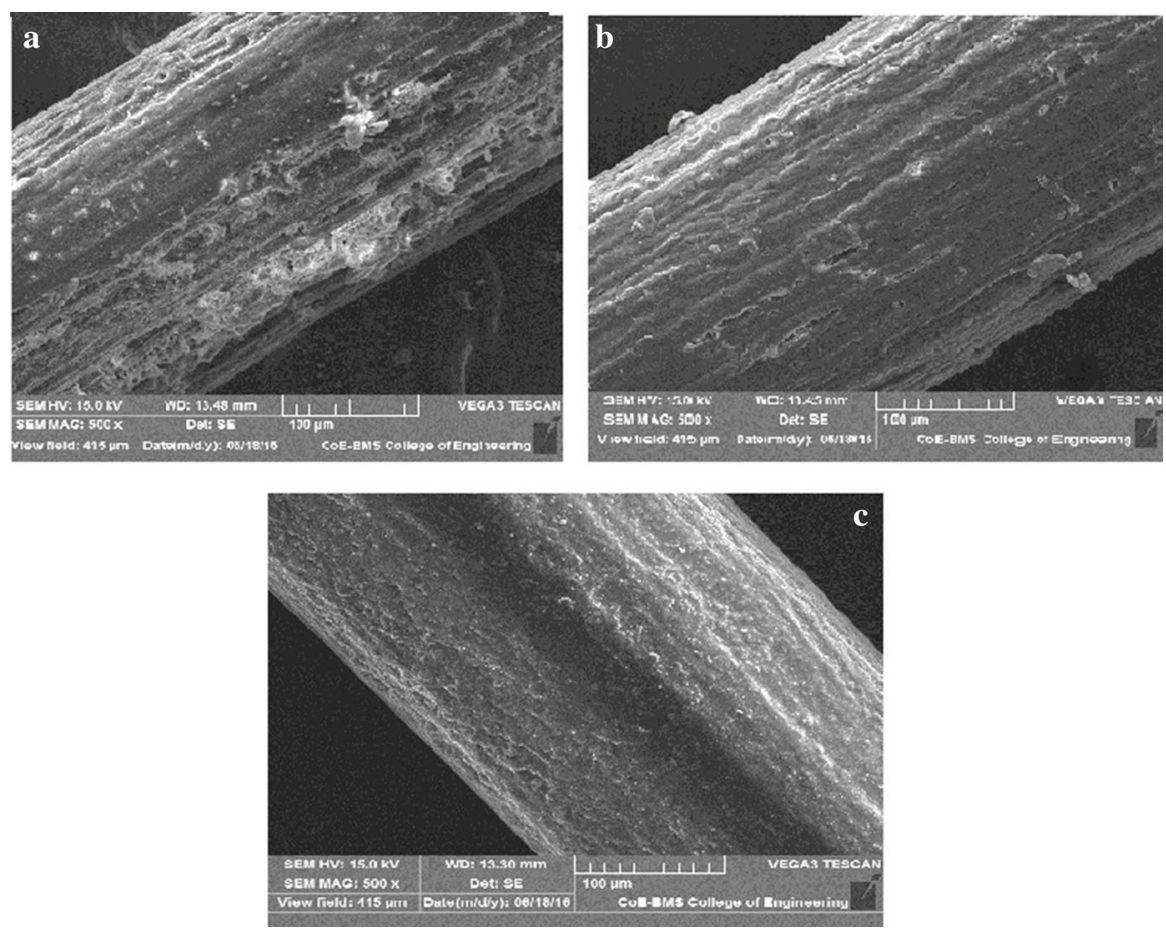

Fig. 4 SEM images of (a) untreated coir fiber, (b) $\mathrm{NaOH}$-treated coir fiber and (c) GA-treated fiber

removal of fatty acids, lignin and impurities present in the fiber. It improves the interaction between fiber and matrix by the formation of mechanical interlocks when it is reinforced in to the matrix for the preparation of composites. Aldehyde-treated fiber with GA shows a smooth surface morphology (Fig. 4c). Crosslinked cellulose and lignin with GA enhance the smoothness of surface of the coir fiber.

\section{Conclusions}

Coir fiber has been chemically treated with the view of manufacturing highperformance natural fiber reinforced composites. Activation energy values showed $\mathrm{NaOH}$ - and GA-treated fibers have better thermal stability. Removal of impurities from the fiber on alkali treatment formed rough surfaces, and crosslinking of both cellulose and lignin on the aldehyde treatment is clearly reflected in the microphotographs of scanning electron microscopy. Such type of treatment improves the stability of coir fiber, and it can be used as effective filler for the preparation of composite materials with excellent physical properties.

Acknowledgements The authors gratefully acknowledge the management of Global Academy of Technology, and Rajarajeswari College of Engineering, Bangalore, for providing the laboratory facilities and their encouragement. 


\section{References}

1. M. Poletto, A.J. Zattera, M.M.C. Forte, R.M.C. Santana, Bioresour. Technol. 109, 148 (2012)

2. K.G.M. Arifuzzaman, A. Md. Shamsul, M. Terano, Indian J. Fibre Text. Res. 37, 20 (2012)

3. S.N. Monteiro, V. Calado, R.J.S. Rodriguez, F.M. Margem, J. Mater. Res. Technol. 1, 117 (2012)

4. M. Ali, J. Civ. Eng. Constr. Technol. 2, 189 (2011)

5. H. Gu, Mater. Des. 30, 3931 (2009)

6. M. Poletto, H.L. Oranghi, A.J. Zattera, Materials 7, 6105 (2014)

7. N. Ezekiel, B.C. Ndazi, C. Nyahumwa, S. Karlsson, Ind. Crops. Prod. 33, 638 (2011)

8. A.I.S. Brígida, V.M.A. Calado, L.R.B. Gonçalves, M.Z. Coelho, Carbohydr. Polym. 79, 832 (2010)

9. M.L. Troëdec, A. Rachini, C. Peyratout, S. Rossignol, E. Max, O. Kaftan, A. Smith, J. Colloid Interface Sci. 356, 303 (2011)

10. E. Abraham, B.L. Deepa, L. Pothen, J. Cintil, S. Thomas, M.J. John, S.S. Narine, Carbohydr. Polym. 92, 1477 (2013)

11. P.J.H. Franco, A.V. González, Compos. Part B Eng. 36, 597 (2005)

12. C. Asasutjarit, S. Charoenvai, J. Hirunlabh, J. Khedari, Compos. Part B Eng. 40, 633 (2009)

13. S. Dixit, P. Verma, Adv. Appl. Sci. Res. 3, 1463 (2012)

14. F.P. Mantia, M.Morreale La, Compos. Part A Appl. Sci. Manuf. 42, 579 (2011)

15. M. Ali, J. Civ. Eng. Constr. Technol. 3, 80 (2012)

16. Z. Xiao, Y. Xie, H. Militz, C. Mai, Holzforschung 64, 475 (2010)

17. Y. Xie, Z. Xiao, T. Grüneberg, H. Militz, C.A.S. Hill, L. Steuernagel, C. Mai, Compos. Sci. Technol. 70, 2003 (2010)

18. D.N. Mahato, B.K. Mathur, S. Bhattacherjee, Indian J. Fibre Text. Res. 20, 202 (1995)

19. L. Segal, J.J. Creely, A.E. Martin Jr., C.M. Conrad, Text. Res. J. 29, 786 (1959)

20. J.I. Langford, A.J.C. Wilson, J. Appl. Crystallogr. 11, 102 (1978)

21. M.C. Popescu, C.M. Popescu, G. Lisa, Y. Sakata, J. Mol. Struct. 988, 65 (2011)

22. A. Johns, M.S. Aan, J. Johns, M.S. Bhagyashekar, C. Nakason, E. Kalkornsurapranee, Iran. Polym. J. 24, 901 (2015)

23. A. Kumar, Y.S. Negi, V. Choudhary, N.K. Bhardwaj, J. Mater. Phys. Chem. 2, 1 (2014)

24. H.L. Ornaghi Jr., M. Poletto, A.J. Zattera, S.C. Amico, Cellulose 21, 177 (2014)

25. D. Chen, D. Lawton, M.R. Thompson, Q. Liu, Carbohydr. Polym. 90, 709 (2012)

26. M. Poletto, A.J. Zattera, R.M.C. Santana, Bioresour. Technol. 126, 7 (2012)

27. V. Tserki, P. Matzinos, S. Kokkou, C. Panayiotou, Compos. Part A 36, 965 (2005) 